\title{
DEVELOPMENT OF PAPER MEMBRANE SWITCHES FOR FULLY FEATURED COMPUTER KEYBOARDS
}

\author{
B. Ramsey, D. Harrison \\ Cleaner Electronics Research \\ Department of Design \\ Brunel University \\ Egham, \\ United Kindom, TW20 OJZ
}

\begin{abstract}
:
This paper documents the successful development and evaluation of a novel membrane switch. The process results in lower environmental burdens and manufacturing costs than current screen-printed polyester solutions.
\end{abstract}

Detailed is the evaluation of offset lithographically printed conductive tracks on paper substrates for application in computer keyboard assemblies. Offset lithographic printing of conductive tracks is an emerging fabrication technique finding application in lowcost, high volume, flexible circuitry applications. The primary advantages of this process are reduced ink volumes, reduced substrate costs and high production speeds, resulting in lower overhead/unit burdens. The use of paper as a substrate material has advantages of lower environmental impact, and lower unit costs than polyester or polycarbonate substrate materials. The selection of a suitable paper is crucial to the conductivity of the printed conductor and lifetime of the switch membrane.

Parameters governing the successful application of new materials to switchpad circuits include track resistance, migration resistance and switch contact resistances. These have been characterised and are reported. Lifetime tests have been conducted and the printed films exposed to a regime of standard $85 R H / 85^{\circ} \mathrm{C}$ and corrosive atmosphere tests. The lifetime of the switches has been investigated, and exceeds the normal industry specification. Several fully featured demonstration keyboards and switch membranes have been constructed and tested. These are currently in use in office environments and will be presented.

Indexing Terms:

Keyboard Switch Membranes, Lithographic Printing, Paper Substrates, Flexible Circuits, Conductive Inks, Lithography.

\section{Introduction}

This paper reports on the application of lithographically printed electrical circuit tracks (Conductive Lithographic Films) and alternative substrate material to membrane switch construction. Conductive Lithographic Films (CLFs) are an emerging additive fabrication technology suitable for manufacturing a variety of electronic circuits and systems. The process employs standard lithographic printing technology and purpose-developed ink pastes to fabricate conductive film patterns on a range of flexible substrates. The technology offers very high production speeds and low material use. Previous work has described Conductive Lithographic Film technology [1], and its application to passive filter networks [2], microwave frequency structures [3], capacitor structures [4],[5], and use with light emitting polymers as electrode structures [6].

\section{Background}

In 1999112 million computers were produced [7]. The requirement for cost effective manufacture in this area is intense. Early keyboards were constructed of individual mechanical switches, but these suffered from short service life. Industry required lower cost higher reliability solutions. The systems developed to fill these requirements fall into two broad categories, Membrane and Pad switches.

Pad switches typically utilise a printed circuit board within the product to carry the conductive traces and to provide mechanical support as the switch is pressed. Both switch contacts are formed on the substrate as part of the etched circuit pattern and protected by a chemically stable, conductive coating such as gold or carbon paste, to prevent corrosion. The switch is activated by pushing a conductive pad onto both contacts, enabling electrical conduction. The pad is often constructed of a carbon loaded rubberised polymer lozenge mounted in a 
rubberised polymer key mat. The form of the key mat determines the tactile characteristics of the key. This type of construction is used extensively in small electronic products such as calculators and telephones.

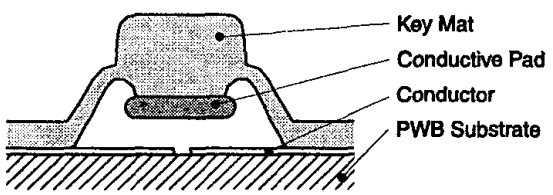

Figure 1: Simplified diagram of a Pad Switch

Larger, more complex keyboards and switch arrays usually utilise membrane switches. These typically consist of a polyester substrate screenprinted with silver paste to form the conductive traces and switch contacts. The substrate is folded back on itself, or faced to another printed sheet, and the conductive traces separated by an insulating spacer. The spacer is typically a polyester sheet with holes punched where the switch contacts need to touch, but may be a thick screen-printed dielectric layer. Activation of the switch is achieved by pressing the two contacts together. Mechanical support is usually achieved by mounting the assembly on part of the product chassis, subassembly or shell moulding.

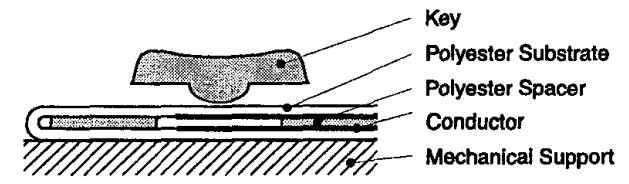

Figure 2: Simplified diagram of a Membrane Switch

In practice, hybrid combinations and variations of these two designs account for the majority of low power mechanical switches in contemporary electronic products.

The electrical characteristic of most importance to membrane switch operation is electrical resistance. The effects of intrinsic capacitance and inductance, though influenced by the track geometry are of no consequence in contemporary membrane switches. The electrical resistance of a switch can be considered to comprise two elements, the line resistance and the contact resistance.

Line resistance is the total resistance of the printed track, leading to and away from the switch contacts. It is determined by the bulk resistivity of the printed ink and the physical geometry of the circuit track. The industry usually simplifies this further by using sheet resistivity (Ohms per square) figures, which assume a constant film thickness and allow easy calculations of line resistance if the length and width are known.

Contact resistance is somewhat more variable, and is influenced by the switch closing pressure, switch contact geometry, switch contact corrosion and contact topography. Switch closing pressure is primarily determined by the force with which the key is depressed, but the pad design and the area of contact the key has with the top membrane will also determine the closing pressure. Corrosion products on the contacting surfaces will have a detrimental effect on contact resistance. These may be limited by the use of conductive barrier coatings, typically carbon paste, if required. For most domestic and office environments barrier coatings are not necessary. Contact topography is largely determined by the ink paste and deposition process, though it will change over the switch life. Typically, the root mean square (RMS) roughness of the printed switch contacts will be several microns $\left(10^{-6} \mathrm{~m}\right)$. Rough contacts have higher resistances than smooth counterparts, as the points of contact are fewer. Contact resistance normally increases as the switch is repeatedly used; an increase of $20 \%$ over 20 million cycles is not untypical [8].

The service life required of a membrane switch is dependent on the application it is used for; Table 1 lists typical specifications.

\begin{tabular}{|l|l|}
\hline Application & Life Cycles \\
\hline Computer Keyboards & $2 \times 10^{7}$ \\
Typewriters & $1 \times 10^{7}$ \\
Copiers \& Telephones & $1 \times 10^{6}$ \\
Calculators & $5 \times 10^{5}$ \\
Toys \& Games & $1 \times 10^{5}$ \\
\hline
\end{tabular}

Table 1: Typical Switch Life Requirements [8]

The likely cause of failure of a membrane switch is usually either electrical or mechanical wear of the switch contact surfaces, or chemical corrosion. It is not often mechanical failure of the substrate or switch mechanics. Mechanical wear of the contact surfaces will occur if the switch contact fragments due to repeated compressive loading.

Excessive electrical wear will result if the switched current is too high. Every switch cycle comprises an instant where the electrical current will pass through an infinitely small contact point. As this occurs the heat energy build up 
resultant of the increased contact resistance can be enough to erode the contact. This may result in lost contact material, or a movement of material from one contact to the other. As a result high voltages across the contacts and high switched currents will rapidly degrade switch life. Corrosion products can cause failure by two mechanisms, contact insulation and migration.

Contact insulation can occur if elements of the ink film react with atmospheric pollutants. Sulphurous gasses can be particularly problematic, though the level found in most modern environments is generally insufficient to cause problems. Careful design of membrane and track geometries has also been employed to reduce exposure.

Migration occurs if a potential gradient and critical quantities of moisture occur between two silver loaded circuit tracks. It is an electrochemical reaction resulting in the growth of conductive silver dendrites between the tracks, eventually resulting in a short circuit. Careful circuit design, minimising steep potential gradients in the same plane, minimises dendrite formation. For product intended for harsh environments it is common practice to overprint silver tracks with carbon pastes. Though these result in a higher contact resistance, they also reduce corrosion-related failures significantly.

The voltage and current a keyboard membrane switch operates at is determined by the circuitry it is in electrical contact with. In most cases this is an encoder IC. The encoder determines which switch on the matrix has been closed and sends the appropriate serial data to the CPU. Encoders present very high impedance to the membrane circuitry. This ensures only minute current flow occurs when the switch contacts are closed, and enables the use of moderately high resistance membrane circuitry. Typical current flow in contemporary computer keyboards is normally less than $0.1 \mathrm{~mA}$, and the potential applied is normally 3-9 volts. The closed switch resistance permissible may be as high as $10 \mathrm{Kohms,}$ though in practice the combined printed track and switch contact resistances are seldom higher than $500 \mathrm{Ohms}$ [8]. Open switch resistance needs to remain higher than the maximum permissible closed switch resistance if the encoder is not to erroneously trigger. In practice open switch resistances are seldom lower than tens of MegaOhms unless there are high levels of moisture present between circuit tracks or migration has occurred.

\section{Method}

To assess the application of CLF circuitry printed on paper for keyboard membrane switches, the screen-printed polyester components of an existing product were replaced. The Cherry ${ }^{\circledR}$ Business Line $₫$ keyboard was selected as it offers a highly optimised design. It is a superb example of integrated design and engineering, minimising componentry, materials, and complexity. In comparison to other keyboards it has far fewer constituent parts and assembles in only a few seconds. Figure 4 illustrates the switch assembly. The rubber spring acts as the key return and determines the tactile feedback the keyboard user experiences. This design enables the mechanical aspects of each key to comprise just three separate parts, two of which are common to the whole product; the product housing, and a rubber spring, the third being each individual key cap. This contrasts to many other designs, which use individual spring and key cap mountings for each key.

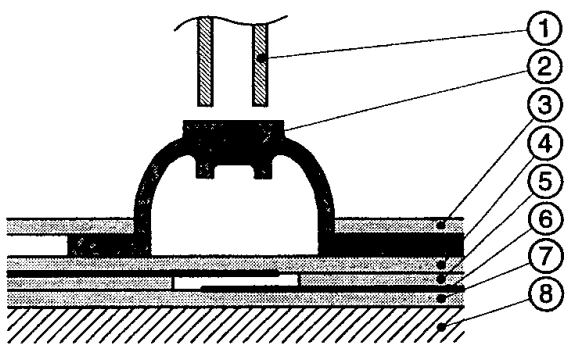

(1) Key Cap

(2) Silicone Rubber spring

(3) Spring Support (paper)

(4) Membrane Switch Substrate, top (paper)

(5) Spacer (paper)

(6) Conductive Circuit (lithographically printed)

(7) Membrane Switch Substrate, bottom (paper)

(8) Mechanical Support (ABS casing)

Figure 4: Simplified diagram of the switch mechanism employed in the cherry $(\mathbb{B}$ keyboard.

The printed paper membranes were prepared using an identical circuit layout to the screenprinted originals. The printed track width was $0.6 \mathrm{~mm}$. The substrate chosen was a standard Gloss Art 80 gsm filled paper, usually used for glossy brochures and magazines. The substrate was imaged using a Heidelberg offset lithographic press, though Roland presses have also been successfully employed. No post printing curing phase was implemented. If it is considered necessary, the sheet resistivity of the printed films can be reduced by about 30 percent by hot air curing at $100^{\circ} \mathrm{C}$ for 5 minutes. 


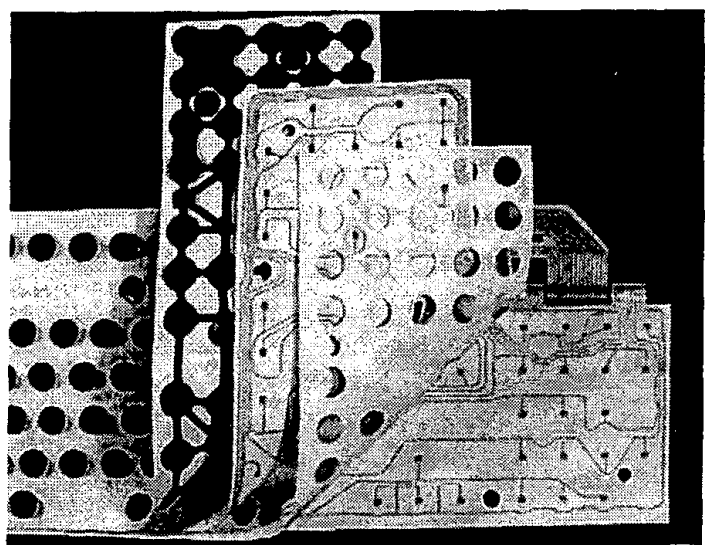

Figure 5: Paper membrane switch constituent parts, left to right: spring support, switch circuitry, spacer, switch circuitry.

Figure 5 shows the three paper components of the keyboard, and the rubber spring matrix. Both of the Membrane Switch Substrates illustrated in Figure 4 are formed from a single piece of material, which is printed, die cut, and folded over so the printed side contacts itself through the holes in the paper spacer (see Figure 6). Also shown are the membrane location pegs.

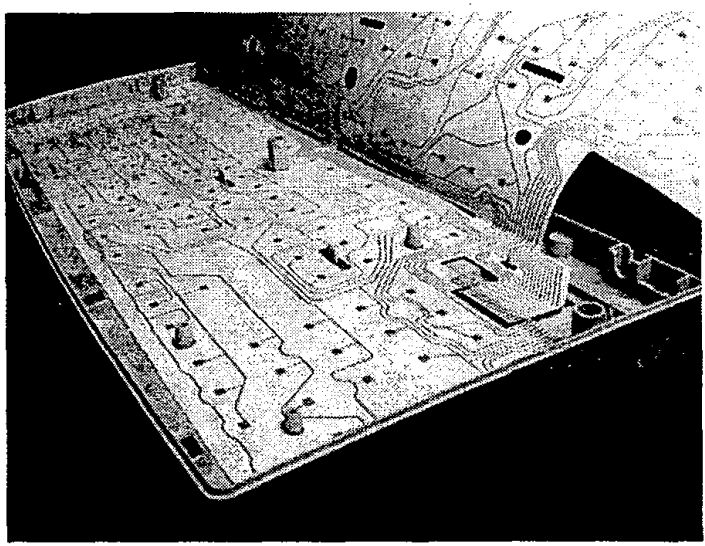

Figure 6: Lithographically printed paper switch membrane.

The Spring Support serves purely mechanical functions, reducing the lateral movement of the rubber spring matrix and locating it accurately between the key cap and membrane switch.

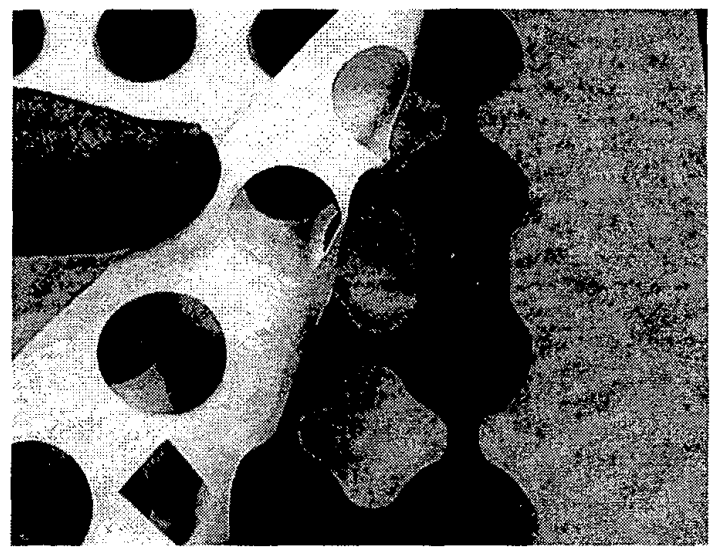

Figure 7: Paper support and rubber spring matrix

The printed paper circuit is connected to the encoder PWB by screwing the PWB into place, trapping the membrane circuitry between the PWB and an elastomer, as illustrated in figure 8. The final assembly is shown in figure 9.

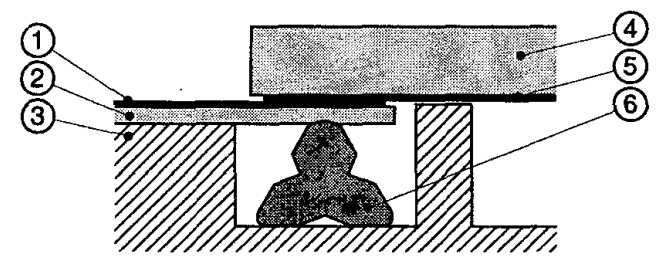
(1) Printed Conductor
(4)FR4 PWB substrate
(2) Paper Substrate
(3) Support (ABS Casing)
(5) Etched Copper circuit
(6) Elastomer

Figure 8: Simplified Membrane Circuit to PWB Connection.

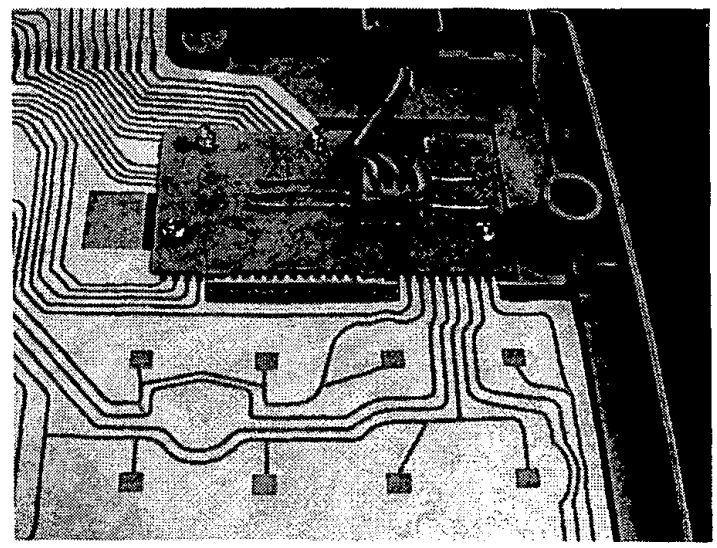

Figure 9: Encoder PWB and paper switch membrane. 
Circuit Track Resistance

The sheet resistivity of the paper membrane switch circuitry is $\approx 150 \mathrm{mOhms} / \mathrm{sq}$. This compares to about $75 \mathrm{mOhms} / \mathrm{sq}$. for the screenprinted original. Resistivity measurements were averaged measurements as detailed in reference [9] obtained using a four-point probe Low OhmMeter. The resultant track resistances are about twice that of the original, just over 400 ohms in the worst case. The contact resistance is also about twice that of the original circuitry. The total switch resistance, worst case scenario, is $\approx 600 \mathrm{ohms}$, comprising contact resistances of $\approx 1 \mathrm{ohm}$, dependent upon key pressure.

\section{Environmental Test}

To assess the longevity of the printed films for typical product lifecycles a series of environmental tests were conducted on unprotected, unbiased samples.

- IEC-68-2-60:pt.2:Ke1995. Flowing Mixed Gas Corrosion (Test Method 1)

- ISE-62-2-67:pt.2:Cy:1996. Damp Heat, Steady State, Accelerated Test. $85^{\circ} \mathrm{C}-85 \%$ Relative Humidity.

- IEC-68-2-3:Ca:1969. Damp Heat, Steady State, $40^{\circ} \mathrm{C}-93 \%$ Relative Humidity.

Results show no catastrophic failure of either the film or the substrate. In all cases any detrimental effect of the test regimes was more than compensated for by additional film curing. As a result resistance of the samples was found to be lower after exposure.

\section{Migration Resistance}

There are as yet no international standards for testing the susceptibility of printed tracks to migration. Extensive discussion of the phenomena may be found in [10], and a more detailed test procedure is detailed in [11]. These tests simulate a very extreme moisture level $(100 \%)$ and high electrical field strengths in comparison to real world domestic environments. Work at Brunel indicates migration resistance of the lithographic ink films and the conventionally manufactured screenprinted films are comparable. Ongoing testing should confirm these results. Full experimental procedure, results, and discussion are available from the Author, and are awaiting publication.

\section{Lifecycle Performance}

The lifecycle performance of membrane switches is normally determined by placing a unit in a mechanical jig, which activates the switch, whilst under electrical load, and increments a counter if a closed circuit was detected. These jigs typically operate at 3 cycles per second and will tolerate 2 consecutive null returns, i.e. open circuit, before terminating the test. The contact life of the CLF paper membrane switch has been tested to 25 million cycles, a figure in excess of most keyboard specifications, without failure. To-date only a single sample has been tested as 25 million cycles takes 3 months to complete!

In addition to the mechanical lifecycle testing, two keyboards have been in use in real world office environments. One of these has been in place for 5 months, so far, without failure.

\section{Conclusions}

This work has demonstrated the suitability of lithographically printed paper membrane switches for computer keyboard applications. Acceptable electrical performance and mechanical longevity has been demonstrated. Paper/cellulose based substrates can achieve acceptable levels of reliability for application in contemporary electronic products. Coupled with the high production speed and low volume/unit ink requirements of offset lithographic printing this fabrication process can offer environmental benefits of:

- Reduced non renewable resource use, as polyester is replaced by paper/cellulose material;

- Lower energy per unit manufacturing cost;

- Reduced raw material use as the quantity of deposited silver conductor is reduced.

\section{Acknowledgements}

The authors would like to thank Gwent Electronic Materials, Malcom Grandis at Tactus (as was), and VCT limited, all of whom have provided resources and technical expertise.

This research has been part funded by the UK EPSRC Electronic Product Design \& Manufacture Initiative.

\section{References}

[1] RAMSEY, B., EVANS, P. \& HARRISON, D. (1997). A Novel Circuit Fabrication Technique Using Offset Lithography, Journal of Electronics Manufacturing. Vol. 7 No.1 pp 63 - 67.

[2] EVANS, P., RAMSEY, B. \& HARRISON D. (1999) Frequency Selective Filter Structures. IEE Electronics Letters, Vol. 35 No.4 Feb. 1999, p.306.

[3] EVANS, P., RAMSEY, B., HARRISON, D.\& SHEPHERD, P.R. (1997). A Novel Lithographic Technology for Microwave Integrated Circuits. I.E.E. Electronics Letters. Vol.33, No.6, March 1997. p483. 
[4] HARREY,P., RAMSEY, B. EVANS, P. \& HARRISON, D. (1999). A novel manufacturing process for capacitors, Eco Design 1999,Proceedings of 1st International Symposium on Environmentally Conscious Design and Inverse Manufacturing 1st Feb. 1999, Tokyo, Japan p842-848,

[5] HARREY,P., RAMSEY, B. EVANS, P. \& HARRISON, D. (1999). Interdigitated Capacitors by Offset Lithography. Journal of Electronics Manufacturing. Accepted for publication.

[6] LOCHUN, D., SAMUEL, I., RAMSEY, B. \& HARRISON, D. (1999) Post - Processing of Conductive Lithographic Films for Electronic Interconnect. Twenty Fourth International Electronics Manufacturing Technology Symposium. $18^{\text {th }}-19^{\text {th }}$ October 1999 , Austin Convention Center, Austin, Texas, USA.

[7] Consumers Driving PC Sales, (2000) Electronics Times, Miller and Freeman UK Itd. 31.1.00 No. 979 pp 8.

[8] Gilleo, K. (1996) Polymer Thick Film ITP pp 7397 ISBN0-442-01220-9

[9] BUEHLER, M.G., THURBER, W.R. (1978) An experimental Study of Various Cross Sheet Resistor Test Structures. J. Electrochemical Soc. - Solid State Technology. 1978125 (4) 645 - 650.

[10] KRUMBEIN, S.J. (1988) Metallic Electromigration Phenomena. IEEE Transactions on Components, Hybrids, and Manufacturing Technology, CHMT-11 (1) 05-15

[11] LIN, J.C., CHUANG, J.U. (1997) Resistance to Silver Electrolytic Migration for Thick-Film Conductors Prepared from Mixed and Alloyed powders of Ag-15Pd and Ag-30Pd. Journal of the Electrochemical Society. 144 (5) $1652-59$. 